\title{
ERGODIC INEQUALITY OF A TWO-PARAMETER INFINITELY-MANY-ALLELES DIFFUSION MODEL
}

\author{
YOUZHOU ZHOU,* McMaster University
}

\begin{abstract}
In this paper three models are considered. They are the infinitely-many-neutral-alleles model of Ethier and Kurtz (1981), the two-parameter infinitely-many-alleles diffusion model of Petrov (2009), and the infinitely-many-alleles model with symmetric dominance Ethier and Kurtz (1998). New representations of the transition densities are obtained for the first two models and the ergodic inequalities are provided for all three models.
\end{abstract}

Keywords: Two-parameter Poisson-Dirichlet distribution; transition density; ergodic inequality

2010 Mathematics Subject Classification: Primary 60J60

Secondary $37 \mathrm{~A} 30$

\section{Introduction}

The Fleming-Viot process is a very important model in population genetics. It can include various evolutionary forces in a single model, such as mutations and selections. Let $E$ be the type space, and $\mathcal{P}(E)$ be the set of probability measures on $E$. The Fleming-Viot process $Z_{t}$ is a $\mathcal{P}(E)$-valued diffusion process with generator,

$$
\begin{aligned}
A F_{f}(\mu)= & \sum_{1 \leq i<j \leq m}\left(\left\langle\Phi_{i j}^{(m)} f, \mu^{m-1}\right\rangle-\left\langle f, \mu^{m}\right\rangle\right)+\left\langle B^{(m)} f, \mu^{m}\right\rangle \\
& +2 \bar{\sigma} \sum_{i=1}^{m}\left(\left\langle K_{i}^{(m)} f, \mu^{m+2}\right\rangle-\left\langle f, \mu^{m}\right\rangle\right)+\bar{\sigma} m\left\langle f, \mu^{m}\right\rangle,
\end{aligned}
$$

where $F_{f}(\mu)=\left\langle f, \mu^{m}\right\rangle, \mu \in \mathcal{P}(E)$, and $f \in \mathcal{B}\left(E^{m}\right)$. Let $\Phi_{i j}^{(m)}$ be the sampling operator, which replaces the $j$ th variable of $f$ by the $i$ th variable. Let $B$ be the mutation operator, generating a Feller semigroup $\left\{T_{t}, t \geq 0\right\}$ defined by a family of transition probabilities $P(t, x, \mathrm{~d} y)(t>0, x \in E)$, and $B^{(m)}$ is the generator of the semigroup

$$
T_{m}(t) f=\int_{E} \ldots \int_{E} f\left(y_{1}, \ldots, y_{m}\right) P\left(t, x_{1}, \mathrm{~d} y_{1}\right) \cdots P\left(t, x_{m}, \mathrm{~d} y_{m}\right) .
$$

Let $K_{i}^{(m)}$ be the selection operator and

$$
K_{i}^{(m)} f=\frac{\bar{\sigma}+\sigma\left(x_{i}, x_{m+1}\right)-\sigma\left(x_{m+1}, x_{m+2}\right)}{2 \bar{\sigma}} f\left(x_{1}, \ldots, x_{m}\right) .
$$

Received 5 July 2013; revision received 9 March 2014.

* Postal address: School of Statistics and Mathematics, Zhongnan University of Economics and Law, 182 South Lake Avenue, East Lake New Technology Development Zone, Wuhan, Hubei, China 430073. Email address: youzhouzhou1984@gmail.com 
Let $\sigma(x, y)$ be a symmetric function called the relative fitness of genotype $\{x, y\}$. Define $\bar{\sigma}$ to be $\sup _{x, y, z}|\sigma(x, y)-\sigma(y, z)|$. For a more comprehensive introduction to the Fleming-Viot process; see [5].

If the mutation operator $B$ of Fleming-Viot process $Z_{t}$ is of the form

$$
B f(x)=\frac{\theta}{2} \int_{E}(f(y)-f(x)) \nu_{0}(\mathrm{~d} x), \quad \theta>0, \nu_{0} \in \mathcal{P}(E),
$$

then, for all $t>0, Z_{t}$ is almost surely of purely atomic measure. Denote the totality of purely atomic measures by $\mathcal{P}_{\mathrm{a}}$. For $\mu \in \mathcal{P}_{\mathrm{a}}$, if we consider the decreasing arrangement of the atomic mass of $\mu$, then we will end up with $\left(x_{1}, x_{2}, \ldots\right)$, which consists of a set

$$
\bar{\nabla}_{\infty}=\left\{\left(x_{1}, x_{2}, \ldots\right) \mid x_{1} \geq x_{2} \geq \cdots \geq 0, \sum_{i=1}^{\infty} x_{i} \leq 1\right\} .
$$

We can define an atomic mapping $\rho: \mathcal{P}(E) \rightarrow \bar{\nabla}_{\infty}$ by mapping $\mu$ to its decreasingly ordered atomic vector $\left(x_{1}, x_{2}, \ldots\right)$. Therefore, $\rho\left(Z_{t}\right)$ is a $\bar{\nabla}_{\infty}$-valued process. The Fleming-Viot process is usually called a labeled model and its atomic process $\rho\left(Z_{t}\right)$ is called an unlabeled model.

If there are only random sampling and mutations involved, then $\rho\left(Z_{t}\right)$ is the infinitely-manyneutral-alleles model [4], denoted by $X_{t}$. The generator of $X_{t}$ is

$$
G=\frac{1}{2} \sum_{i, j=1}^{\infty} x_{i}\left(\delta_{i, j}-x_{j}\right) \frac{\partial^{2}}{\partial x_{i} \partial x_{j}}-\frac{\theta}{2} \sum_{i=1}^{\infty} x_{i} \frac{\partial}{\partial x_{i}}, \quad x \in \bar{\nabla}_{\infty} .
$$

If we include selection as well, then the unlabeled model is usually non-Markovian. But if we consider selection of symmetric dominance introduced in [13], then the unlabeled model is a Markov process. We denote this unlabeled model by $X_{t}^{\sigma}$ and call it infinitely-many-alleles diffusion with symmetric dominance; see [6]. The generator of $X_{t}^{\sigma}$ is

$$
G_{\sigma}=G+\sigma \sum_{i=1}^{\infty} x_{i}\left(x_{i}-\varphi_{2}(x)\right) \frac{\partial}{\partial x_{i}}, \quad x \in \bar{\nabla}_{\infty},
$$

where $\varphi_{2}(x)=\sum_{i=1}^{\infty} x_{i}^{2}$, and is called homozygosity in population genetics.

Both $X_{t}$ and $X_{t}^{\sigma}$ are reversible diffusions and have unique stationary distributions. The stationary distribution of $X_{t}$ is the Poisson-Dirichlet distribution $\operatorname{PD}(\theta)$, and the stationary distribution of $X_{t}^{\sigma}$ is

$$
\pi_{\sigma}(\mathrm{d} x)=C_{\sigma} \operatorname{Exp}\left\{\sigma \varphi_{2}(x)\right\} \operatorname{PD}(\theta)(\mathrm{d} x),
$$

where $C_{\sigma}$ is a normalized constant.

Moreover, there is a two-parameter generalization of the $\operatorname{PD}(\theta)$. We call it a two-parameter Poisson-Dirichlet distribution (see [7]) $\mathrm{PD}(\theta, \alpha), \theta+\alpha>0,0<\alpha<1$. Correspondingly, there is a two-parameter generalization [8], [11] of $X_{t}$, denoted by $X_{t}^{\theta, \alpha}$ and called a two-parameter infinitely-many-alleles diffusion model. The two-parameter Poisson-Dirichlet distribution $\operatorname{PD}(\theta, \alpha)$ is the associated stationary distribution. The generator of $X_{t}^{\theta, \alpha}$ is

$$
G^{\theta, \alpha}=\frac{1}{2} \sum_{i, j=1}^{\infty} x_{i}\left(\delta_{i, j}-x_{j}\right) \frac{\partial^{2}}{\partial x_{i} \partial x_{j}}-\frac{1}{2} \sum_{i=1}^{\infty}\left(\theta x_{i}+\alpha\right) \frac{\partial}{\partial x_{i}}, \quad x \in \bar{\nabla}_{\infty} .
$$

However, $X_{t}^{\theta, \alpha}$ has no biological interpretation at all. Whether its labeled model exists is still open. 
In [3], the transition probability of the neutral Fleming-Viot process $Z_{t}$ is obtained. In [2], the transition density function of the unlabeled neutral process $X_{t}$ is also obtained. Therefore, its explicit transition probability is available as well. We can actually obtain the transition probabilities of $X_{t}$ through the transition probabilities of $Z_{t}$; see [7]. In [9], the transition density function of $X_{t}^{\theta, \alpha}$ is obtained as well. In this paper we reorganize the transition density functions of $X_{t}^{\theta, \alpha}$ and obtain a new representation of the transition density functions of $X_{t}^{\theta, \alpha}$. Interestingly, the associated transition probabilities resemble the structure of the transition probabilities for the neutral Fleming-Viot process. This can actually shed some light on the construction of the labeled model of $X_{t}^{\theta, \alpha}$.

Furthermore, the ergodic inequalities of $Z_{t}$ and $X_{t}$ are both available, but similar ergodic inequalities of $X_{t}^{\theta, \alpha}$ and $X_{t}^{\sigma}$ are still missing. In this paper we will obtain the ergodic inequalities of $X_{t}^{\theta, \alpha}$ and $X_{t}^{\sigma}$. It turns out that $X_{t}^{\theta, \alpha}$ and $X_{t}$ share the same ergodic inequality. Lastly, the ergodic inequality of $X_{t}^{\sigma}$ is stronger than the ergodic theorem stated in [6].

The remainder of this paper is organized as follows. In Section 2 we will consider the transition density functions of $X_{t}^{\theta, \alpha}$. In Section 3 we will discuss the ergodic inequalities of $X_{t}^{\theta, \alpha}$ and $X_{t}^{\sigma}$.

\section{The transition density functions of $X_{t}^{\theta, \alpha}$}

In [2] and [9], the explicit transition densities of $X_{t}$ and $X_{t}^{\theta, \alpha}$ are obtained, respectively, through eigen expansion. By making use of these known transition densities, we obtain a new representation.

Theorem 2.1. It holds that $X_{t}^{\theta, \alpha}$ has the following transition density:

$$
p^{\theta, \alpha}(t, x, y)=d_{0}^{\theta}(t)+d_{1}^{\theta}(t)+\sum_{n=2}^{\infty} d_{n}^{\theta}(t) p_{n}^{\theta, \alpha}(x, y),
$$

where

$$
\begin{aligned}
& d_{n}^{\theta}(t)=\sum_{m=n}^{\infty} \frac{2 m+\theta-1}{m !}(-1)^{m-n}\left(\begin{array}{c}
m \\
n
\end{array}\right)(n+\theta)_{(m-1)} \mathrm{e}^{-\lambda_{m} t}, \quad n \geq 1, \\
& d_{0}^{\theta}(t)=1-\sum_{m=1}^{\infty} \frac{2 m+\theta-1}{m !}(-1)^{m-1} \theta_{(m-1)} \mathrm{e}^{-\lambda_{m} t}, \\
& \lambda_{1}=0, \lambda_{m}=\frac{m(m-1+\theta)}{2}, \quad m \geq 2 .
\end{aligned}
$$

Moreover,

$$
p_{n}^{\theta, \alpha}(x, y)=\sum_{|\eta|=n} \frac{p_{\eta}(x) p_{\eta}(y)}{\int p_{\eta} d \operatorname{PD}(\theta, \alpha)}
$$

$\eta=\left(\eta_{1}, \ldots, \eta_{l}\right)$ is a partition of $n$ and $|\eta|=\sum_{i=1}^{l} \eta_{i}$.

Define $a_{i}(\eta)=\#\left\{j \mid \eta_{j}=i, 1 \leq j \leq l\right\}$. Then $p_{\eta}(x)$ is the continuous extension of

$$
\frac{n !}{\eta_{1} ! \ldots \eta_{l} ! a_{1}(\eta) ! \ldots a_{n}(\eta) !} \sum_{i_{1}, \ldots, i_{l} \text { distinct }} x_{i_{1}}^{\eta_{1}} \ldots x_{i_{l}}^{\eta_{l}} .
$$


Proof. Due to [9], the transition density of $X_{t}^{\theta, \alpha}$ is

$$
p^{\theta, \alpha}(t, x, y)=1+\sum_{m=2}^{\infty} \mathrm{e}^{-\lambda_{m} t} Q_{m}^{\theta, \alpha}(x, y) .
$$

Moreover, for $\theta>-\alpha, 0<\alpha<1$, there exist constants $c$ and $d$, such that

$$
Q_{m}^{\theta, \alpha}(x, y) \leq\left(\mathrm{cm}^{d}\right)^{m} .
$$

Therefore, for $t_{0}>0$, and for all $t \in\left[t_{0}, \infty\right)$, we have

$$
\begin{aligned}
\sum_{m=2}^{\infty} \operatorname{Exp}\left\{-\lambda_{m} t\right\} Q_{m}^{\theta, \alpha}(x, y) & \leq \sum_{m=2}^{\infty} \operatorname{Exp}\left\{-\lambda_{m} t\right\}\left(c m^{d}\right)^{m} \\
& =\sum_{m=2}^{\infty}\left(\frac{c m^{d}}{\exp (t(m+\theta-1) / 2)}\right)^{m} \\
& \leq \sum_{m=2}^{\infty}\left(\frac{c m^{d}}{\exp \left(t_{0}(m+\theta-1) / 2\right)}\right)^{m} .
\end{aligned}
$$

Since $\lim _{m \rightarrow+\infty} c m^{d} / \exp \left(t_{0}(m+\theta-1) / 2\right)=0$, the exists $M>0$ such that, for all $m>M$,

$$
\frac{c m^{d}}{\exp \left(t_{0}(m+\theta-1) / 2\right)}<\frac{1}{2} \text {. }
$$

Because $\sum_{m \geq 1} 1 / 2^{m}$ is convergent; then, by Weierstrass's M-test, $p^{\theta, \alpha}(t, x, y)$ is uniformly convergent on $\left[t_{0},+\infty\right) \times \bar{\nabla}_{\infty} \times \bar{\nabla}_{\infty}$, and, thus, is continuous. Next, by Fubini's theorem, we can rearrange $p^{\theta, \alpha}(t, x, y)$ by switching the order of summation. Then

$$
\begin{aligned}
p^{\theta, \alpha}(t, x, y)=1+\sum_{m=2}^{\infty} \mathrm{e}^{-\lambda_{m} t}( & \sum_{n=2}^{m} \frac{2 m+\theta-1}{m !}(-1)^{m-n}\left(\begin{array}{c}
m \\
n
\end{array}\right)(n+\theta)_{(m-1)} p_{n}^{\theta, \alpha}(x, y) \\
& +\frac{2 m+\theta-1}{m !}(-1)^{m-1}(\theta+1)_{(m-1)} m p_{1}^{\theta, \alpha}(x, y) \\
& \left.+\frac{2 m+\theta-1}{m !}(-1)^{m} \theta_{(m-1)} p_{0}^{\theta, \alpha}(x, y)\right) .
\end{aligned}
$$

Since $p_{1}^{\theta, \alpha}(x, y), p_{0}^{\theta, \alpha}(x, y)=1$, we have

$$
\begin{aligned}
p^{\theta, \alpha}(t, x, y)= & 1+\sum_{m=2}^{\infty} \mathrm{e}^{-\lambda_{m} t} \sum_{n=2}^{m} \frac{2 m+\theta-1}{m !}(-1)^{m-n}\left(\begin{array}{c}
m \\
n
\end{array}\right)(n+\theta)_{(m-1)} p_{n}^{\theta, \alpha}(x, y) \\
& +\sum_{m=2}^{\infty} \mathrm{e}^{-\lambda_{m} t}\left(\frac{2 m+\theta-1}{m !}(-1)^{m-1}(\theta+1)_{(m-1)} m\right. \\
& \left.+\frac{2 m+\theta-1}{m !}(-1)^{m} \theta_{(m-1)}\right) \\
= & 1+\sum_{m=2}^{\infty} \mathrm{e}^{-\lambda_{m} t} \sum_{n=2}^{m} \frac{2 m+\theta-1}{m !}(-1)^{m-n}\left(\begin{array}{c}
m \\
n
\end{array}\right)(n+\theta)_{(m-1)} p_{n}^{\theta, \alpha}(x, y) \\
& +\sum_{m=2}^{\infty} \mathrm{e}^{-\lambda_{m} t} \frac{2 m+\theta-1}{m !}(-1)^{m-1}\left[m(\theta+1)_{(m-1)}-\theta_{(m-1)}\right] .
\end{aligned}
$$


When $m=1, m(\theta+1)_{(m-1)}-\theta_{(m-1)}=0$. Then we have

$$
\begin{aligned}
p^{\theta, \alpha}(t, x, y)= & 1-\sum_{m=1}^{\infty} \mathrm{e}^{-\lambda_{m} t} \frac{2 m+\theta-1}{m !}(-1)^{m-1} \theta_{(m-1)} \\
& +\sum_{m=1}^{\infty} \mathrm{e}^{-\lambda_{m} t} \frac{2 m+\theta-1}{m !}(-1)^{m-1} m(\theta+1)_{(m-1)} \\
& +\sum_{m=2}^{\infty} \mathrm{e}^{-\lambda_{m} t} \sum_{n=2}^{m} \frac{2 m+\theta-1}{m !}(-1)^{m-n}\left(\begin{array}{c}
m \\
n
\end{array}\right)(n+\theta)_{(m-1)} p_{n}(x, y) \\
= & d_{0}^{\theta}(t)+d_{1}^{\theta}(t) \\
& +\sum_{m=2}^{\infty} \mathrm{e}^{-\lambda_{m} t} \sum_{n=2}^{m} \frac{2 m+\theta-1}{m !}(-1)^{m-n}\left(\begin{array}{c}
m \\
n
\end{array}\right)(n+\theta)_{(m-1)} p_{n}^{\theta, \alpha}(x, y) .
\end{aligned}
$$

Let us switch the order of summation. Then we have

$$
p^{\theta, \alpha}(t, x, y)=d_{0}^{\theta}(t)+d_{1}^{\theta}(t)+\sum_{n=2}^{+\infty} d_{n}^{\theta}(t) p_{n}^{\theta, \alpha}(x, y) .
$$

Define $v_{n}^{\theta, \alpha}(x, \mathrm{~d} y)=p_{n}^{\theta, \alpha}(x, y) \operatorname{PD}(\theta, \alpha)(\mathrm{d} y)$, then the transition probability of $X_{t}^{\theta, \alpha}$ is

$$
P^{\theta, \alpha}(t, x, \mathrm{~d} y)=\left(d_{0}^{\theta}(t)+d_{1}^{\theta}(t)\right) \operatorname{PD}(\theta, \alpha)(\mathrm{d} y)+\sum_{n=2}^{\infty} d_{n}^{\theta}(t) v_{n}^{\theta, \alpha}(x, \mathrm{~d} y) .
$$

Remark 2.1. The transition probability of $X_{t}$ also has the same structure as (2.1). Moreover, since $X_{t}$ has an entrance boundary $\bar{\nabla}_{\infty}-\nabla_{\infty}$, i.e. $X_{t}$ immediately moves into $\nabla_{\infty}$ and never exits regardless of its starting point. S.N. Ethier informed the author that a similar result can also be obtained for $X_{t}^{\theta, \alpha}$.

For both $X_{t}$ and $X_{t}^{\theta, \alpha}$, the coefficients $d_{n}^{\theta}(t), n \geq 0$, are the same. When $\theta \geq 0$, they are the distributions of the ancestral process discussed by Tavaré [12]. However, for $X_{t}^{\theta, \alpha}, \theta$ could be negative and $d_{n}^{\theta}(t)$ is not a probability distribution anymore. However, if we collapse the states 0 and 1 , and relabel it as 1 , then $d_{1}^{\theta}(t)+d_{0}^{\theta}(t), d_{n}^{\theta}(t), n \geq 2$, define a probability distribution. We can generalize this structure to the case where $\theta>-1$. The estimation of the tail probability obtained in [12] is still true when $\theta>-1$.

Proposition 2.1. For $\theta>-1$, we have

$$
\mathrm{e}^{-\lambda_{n} t} \leq \sum_{k=n}^{\infty} d_{k}^{\theta}(t) \leq \frac{(n+\theta)(n)}{n_{[n]}} \mathrm{e}^{-\lambda_{n} t}
$$

In particular, when $n=2$, we have

$$
\sum_{k=2}^{\infty} d_{k}^{\theta}(t) \leq \frac{(2+\theta)(3+\theta)}{2} \mathrm{e}^{-(\theta+1) t}
$$


Proof. Consider a pure-death Markov chain $B_{t}$ in $\{1,2, \ldots, m\}$ with Q matrix,

$$
Q=\left(\begin{array}{cccccc}
0 & 0 & 0 & \cdots & 0 & 0 \\
\lambda_{2} & -\lambda_{2} & 0 & \cdots & 0 & 0 \\
0 & \lambda_{3} & -\lambda_{3} & \cdots & 0 & 0 \\
\vdots & \vdots & \vdots & \cdots & \vdots & \vdots \\
0 & 0 & 0 & \cdots & \lambda_{m} & -\lambda_{m}
\end{array}\right)
$$

where $\lambda_{k}=\frac{1}{2} k(k+\theta-1), k \geq 2$. Following the similar arguments in Theorem 4.3 of [7], we will be able to find all the left eigenvectors and right eigenvectors of $Q$. Denote the matrix consisting of left eigenvectors by $U=\left(u_{i j}\right)$ and the matrix consisting of right eigenvectors by $V=\left(v_{i j}\right)$, where

$$
u_{i j}= \begin{cases}\delta_{1 j}, & i=1, \\
0, & j>i>1, \\
(-1)^{i-j}\left(\begin{array}{l}
i \\
j
\end{array}\right) \frac{(j+\theta)_{(i-1)}}{(i+\theta)_{(i-1)}}, & j \leq i, i>1,\end{cases}
$$

and

$$
v_{i j}= \begin{cases}1, & j=1, \\
0, & j>i, \\
\left(\begin{array}{l}
i \\
j
\end{array}\right) \frac{(j+\theta)_{(j)}}{(i+\theta)_{(j)}}, & 1<j \leq i .\end{cases}
$$

Note that the row vectors of $U$ are the left eigenvectors of $Q$ and the column vectors of $V$ are the right eigenvectors of $Q$. Similarly, we can also show that $U V=I$ and $Q$ is diagonlized as $V \Lambda U$, where $\Lambda=\operatorname{diag}\left\{0,-\lambda_{2}, \ldots,-\lambda_{m}\right\}$. Therefore, the transition matrix $P_{t}$ is

$$
P_{t}=\mathrm{e}^{t Q}=V \mathrm{e}^{\Lambda t} U
$$

By direct computation, we know that, for $2 \leq n \leq m$,

$$
P_{m n}(t)=\sum_{k=n}^{m}(-1)^{k-n}\left(\begin{array}{l}
m \\
k
\end{array}\right)\left(\begin{array}{l}
k \\
n
\end{array}\right) \frac{(\theta+k)_{(k)}}{(\theta+m)_{(k)}} \frac{(\theta+n)_{(k-1)}}{(\theta+k)_{(k-1)}} \mathrm{e}^{-\lambda_{k} t} .
$$

Letting $m \rightarrow+\infty$, we have $d_{n}^{\theta}(t)=\lim _{m \rightarrow \infty} P_{m n}(t)$.

The remaining arguments are essentially due to Tavaré.

By the martingale argument in Chapter 6 of [10], we know that

$$
Z_{n}(t)=\frac{\mathrm{e}^{\lambda_{n} t}\left(B_{t}\right)_{[n]}}{\left(B_{t}+\theta\right)_{(n)}},
$$

because $\mathrm{e}^{-\lambda_{n} t}$ is one eigenvalue of $P_{t}$ and

$$
\left(0,0, \ldots, 0, \frac{n_{[n]}}{(n+\theta)_{(n)}}, \ldots, \frac{k_{[n]}}{(k+\theta)_{(n)}}, \ldots, \frac{m_{[n]}}{(m+\theta)_{(n)}}\right)^{\top}
$$

is the corresponding eigenvector. So,

$$
E Z_{n}(t)=Z_{n}(0)=\frac{m_{[n]}}{(m+\theta)_{(n)}} .
$$


Since, for $n \leq k \leq m$,

$$
\frac{n_{[n]}}{(n+\theta)_{(n)}} \leq \frac{k_{[n]}}{(k+\theta)_{(n)}} \leq \frac{m_{[n]}}{(m+\theta)_{(n)}}
$$

and

$$
\frac{\mathrm{e}^{-\lambda_{n} t} m_{[n]}}{(m+\theta)_{(n)}}=\mathrm{e}^{-\lambda_{n} t} E Z_{n}(t)=\sum_{k=n}^{m} \frac{k_{[n]}}{(k+\theta)_{(n)}} P_{m k}(t)
$$

we have

$$
\frac{n_{[n]}}{(n+\theta)_{(n)}} P\left(B_{t} \geq n \mid B_{0}=m\right) \leq \frac{\mathrm{e}^{-\lambda_{n} t} m_{[n]}}{(m+\theta)_{(n)}} \leq \frac{m_{[n]}}{(m+\theta)_{(n)}} P\left(B_{t} \geq n \mid B_{0}=m\right) .
$$

Thus, we have

$$
\mathrm{e}^{-\lambda_{n} t} \leq P\left(B_{t} \geq n \mid B_{0}=m\right) \leq \frac{(n+\theta)_{(n)}}{n_{[n]}} \mathrm{e}^{-\lambda_{n} t} .
$$

Letting $m \rightarrow \infty$, we have

$$
\mathrm{e}^{-\lambda_{n} t} \leq \sum_{k=n}^{\infty} d_{k}^{\theta}(t) \leq \frac{(n+\theta)_{(n)}}{n_{[n]}} \mathrm{e}^{-\lambda_{n} t}
$$

\section{Ergodic inequalities}

By making use of the transition probability (2.1) and the estimation of the tail probability (2.2), we can easily obtain the following ergodic inequality of $X_{t}^{\theta, \alpha}$.

Theorem 3.1. For $X_{t}^{\theta, \alpha}$, we have the ergodic inequality

$$
\sup _{x \in \bar{\nabla}_{\infty}}\left\|P^{\theta, \alpha}(t, x, \cdot)-\operatorname{PD}(\theta, \alpha)(\cdot)\right\|_{\text {var }} \leq \frac{(2+\theta)(3+\theta)}{2} \operatorname{Exp}\{-(\theta+1) t\}, \quad t \geq 0 .
$$

Proof. Denote $\mathcal{B}$ to be the totality of Borel subsets of $\bar{\nabla}_{\infty}$. Then

$$
\begin{aligned}
\left\|P^{\theta, \alpha}(t, x, \cdot)-\operatorname{PD}(\theta, \alpha)(\cdot)\right\|_{\mathrm{var}}= & \sup _{A \in \mathscr{B}}\left|P^{\theta, \alpha}(t, x, A)-\operatorname{PD}(\theta, \alpha)(A)\right| \\
= & \sup _{A \in \mathscr{B}} \mid\left(d_{0}^{\theta}(t)+d_{1}^{\theta}(t)\right) \operatorname{PD}(\theta, \alpha)(A) \\
& +\sum_{n=2}^{\infty} d_{n}^{\theta}(t) v_{n}^{\theta, \alpha}(A)-\operatorname{PD}(\theta, \alpha)(A) \mid \\
= & \sup _{A \in \mathcal{B}}\left|\sum_{n=2}^{\infty} d_{n}^{\theta}(t)\left(v_{n}^{\theta, \alpha}(A)-\operatorname{PD}(\theta, \alpha)(A)\right)\right| \\
\leq & \sum_{n=2}^{\infty} d_{n}^{\theta}(t) \sup _{A \in \mathscr{B}}\left|v_{n}^{\theta, \alpha}(A)-\operatorname{PD}(\theta, \alpha)(A)\right| \\
\leq & \sum_{n=2}^{\infty} d_{n}^{\theta}(t) \leq \frac{(\theta+2)(\theta+3)}{2} \mathrm{e}^{-(\theta+1) t} .
\end{aligned}
$$


Remark 3.1. As can be seen, the ergodic inequality of $X_{t}^{\theta, \alpha}$ is the same as the ergodic inequality of $X_{t}$ obtained in [2]. But for $X_{t}^{\theta, \alpha}, \theta$ could be negative.

Since $X_{t}^{\sigma}$ is absolutely continuous with respect to $X_{t}, \bar{\nabla}_{\infty}-\nabla_{\infty}$ should also serve as an entrance boundary of $X_{t}^{\sigma}$. Hence, we can change the value of the density function $p_{\sigma}(t, x, y)$ when $x$ or $y$ is in $\bar{\nabla}_{\infty}-\nabla_{\infty}$. Therefore, $p_{\sigma}(t, x, y)$ can be chosen to be the continuous extension of $\left.p_{\sigma}(t, x, y)\right|_{\nabla_{\infty} \times \nabla_{\infty}}$. Moreover, $p_{\sigma}(t, x, y)$ is symmetric because $X_{t}^{\sigma}$ is reversible. As stated in [9], the Poincaré inequality of $X_{t}^{\sigma}$ also holds. Therefore, it guarantees the $L_{2}$-exponential convergence of $X_{t}^{\sigma}$. By running the argument in Theorem 8.8 of [1], we can also obtain the following ergodic inequality.

Theorem 3.2. For $X_{t}^{\sigma}$, there exists $K(\theta, \sigma)$, such that

$$
\sup _{x \in \bar{\nabla} \infty}\left\|P^{\sigma}(t, x, \cdot)-\pi_{\sigma}(\cdot)\right\|_{\mathrm{var}} \leq K(\theta, \sigma) \operatorname{Exp}\left\{-\operatorname{gap}\left(G_{\sigma}\right) t\right\}, \quad t \geq 0 .
$$
Since

Proof. We will follow the argument in Theorem 8.8 of [1]. Define $\mu^{x}(\cdot)=P_{x}\left(X_{s}^{\sigma} \in \cdot\right)$.

$$
P^{\sigma}(t, x, \cdot)=\int_{\bar{\nabla}_{\infty}} P^{\sigma}(t-s, z, \cdot) P^{\sigma}(s, x, \mathrm{~d} z),
$$

we have

$$
P^{\sigma}(t, x, \cdot)=\mu^{x} P_{t-s}^{\sigma}(\cdot)
$$

Therefore,

$$
\left\|P^{\sigma}(t, x, \cdot)-\pi(\cdot)\right\|_{\mathrm{var}}=\left\|\mu^{x} P_{t-s}^{\sigma}(\cdot)-\pi(\cdot)\right\|_{\mathrm{var}} .
$$

By part (1) in Theorem 8.8 of [1], we have, for all $t \geq s$,

$$
\begin{aligned}
\left\|P^{\sigma}(t, x, \cdot)-\pi(\cdot)\right\|_{\text {var }} & \leq\left\|\frac{\mathrm{d} \mu^{x}}{\mathrm{~d} \pi_{\sigma}}-1\right\|_{2} \mathrm{e}^{-(t-s) \operatorname{gap}\left(G_{\sigma}\right)} \\
& =\sqrt{\int p_{\sigma}(s, x, y)^{2} \pi_{\sigma}(\mathrm{d} y)-1 \mathrm{e}^{-(t-s) \operatorname{gap}\left(G_{\sigma}\right)} .}
\end{aligned}
$$

Therefore, for $t \geq s$, we have

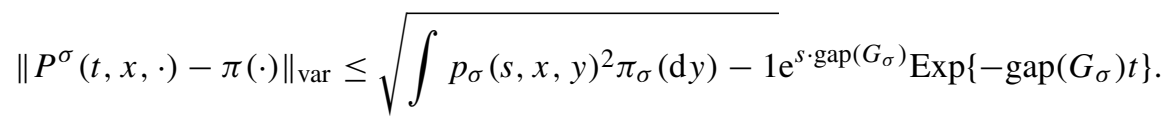

Due to (4.17) of [6] and Theorem 3.3 of [9], we can conclude that there exists a constant $D(\sigma, t)>0$, such that

$$
p_{\sigma}(t, x, y) \leq D(\sigma, t)
$$

If we choose $s=\frac{1}{2}$, the constant

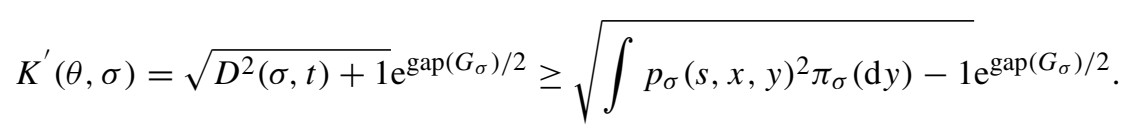

Then we have

$$
\sup _{x \in \bar{\nabla} \infty}\left\|P^{\sigma}(t, x, \cdot)-\pi_{\sigma}(\cdot)\right\|_{\mathrm{var}} \leq K^{\prime}(\theta, \sigma) \operatorname{Exp}\left\{-\operatorname{gap}\left(G_{\sigma}\right) t\right\}, \quad \text { for all } t \geq \frac{1}{2} .
$$


Moreover,

$$
\sup _{x \in \bar{\nabla}_{\infty}}\left\|P^{\sigma}(t, x, \cdot)-\pi_{\sigma}(\cdot)\right\|_{\mathrm{var}} \leq 1, \quad \text { for all } t \geq 0 .
$$

Thus, for all $t \in\left[0, \frac{1}{2}\right]$, if we choose $K^{\prime \prime}(\theta, \sigma)$ such that

$$
K^{\prime \prime}(\theta, \sigma) \mathrm{e}^{-\operatorname{gap}\left(G_{\sigma}\right) / 2} \geq 1,
$$

then, for all $t \in\left[0, \frac{1}{2}\right]$,

$$
\sup _{x \in \bar{\nabla}_{\infty}}\left\|P^{\sigma}(t, x, \cdot)-\pi_{\sigma}(\cdot)\right\|_{\mathrm{var}} \leq 1 \leq K^{\prime \prime}(\theta, \sigma) \mathrm{e}^{-\operatorname{gap}\left(G_{\sigma}\right) / 2} \leq K^{\prime \prime}(\theta, \sigma) \operatorname{Exp}\left\{-\operatorname{gap}\left(G_{\sigma}\right) t\right\} .
$$

Therefore, choosing $K(\theta, \sigma)=\max \left\{K^{\prime}(\theta, \sigma), K^{\prime \prime}(\theta, \sigma)\right\}$, we have

$$
\sup _{x \in \bar{\nabla}_{\infty}}\left\|P^{\sigma}(t, x, \cdot)-\pi_{\sigma}(\cdot)\right\|_{\mathrm{var}} \leq K(\theta, \sigma) \operatorname{Exp}\left\{-\operatorname{gap}\left(G_{\sigma}\right) t\right\} .
$$

Remark 3.2. Presumably, we could have an ergodic inequality of $X_{t}^{\sigma}$ if we apply Theorem 8.8 of [1]. But due to the special property of $X_{t}^{\sigma}$, this theorem is actually a refinement of the ergodic inequality deduced from Theorem 8.8 of [1]. Furthermore, this theorem is stronger than the ergodic theorem of [6].

\section{Acknowledgements}

This paper is part of my $\mathrm{PhD}$ work. I am so grateful for the support of my $\mathrm{PhD}$ advisor, Shui Feng. Moreover, I would also like to thank Stewart N. Ethier for his suggestion in the proof of proposition 2.1 .

\section{References}

[1] Chen, M.-F. (2005). Eigenvalues, Inequalities, and Ergodic Theory. Springer, London.

[2] Ethier, S. N. (1992). Eigenstructure of the infinitely-many-neutral-alleles diffusion model. J. Appl. Prob. 29, 487-498.

[3] Ethier, S. N. And Griffiths, R. C. (1993). The transition function of a Fleming-Viot process. Ann. Prob. 21, 1571-1590.

[4] Ethier, S. N. And Kurtz, T. G. (1981). The infinitely-many-neutral-alleles diffusion model. Adv. Appl. Prob. 13, 429-452.

[5] Ethier, S. N. and Kurtz, T. G. (1993). Fleming-Viot processes in population genetics. SIAM J. Control Optimization 31, 345-386.

[6] Ethier, S. N. And Kurtz, T. G. (1998). Coupling and ergodic theorems for Fleming-Viot processes. Ann. Prob. 26, 533-561.

[7] Feng, S. (2010). The Poisson-Dirichlet Distribution and Related Topics. Models and Asymptotic Behaviors. Springer, Heidelberg.

[8] Feng, S. And Sun, W. (2010). Some diffusion processes associated with two parameter Poisson-Dirichlet distribution and Dirichlet process. Prob. Theory Relat. Fields 148, 501-525.

[9] Feng, S., Sun, W., WANG, F.-Y. And Xu, F. (2011). Functional inequalities for the two-parameter extension of the infinitely-many-neutral-alleles diffusion. J. Funct. Anal. 260, 399-413.

[10] Karlin, S. and Taylor, H. M. (1981). A Second Course in Stochastic Processes. Academic Press, New York.

[11] Petrov, L. A. (2009). A two-parameter family of infinite-dimensional diffusions on the Kingman simplex. Funct. Anal. Appl. 43, 279-296.

[12] TAvaré, S. (1984). Line-of-descent and genealogical processes, and their applications in population genetics models. Theoret. Pop. Biol. 26, 119-164.

[13] Watterson, G. A. (1977). Heterosis or neutrality? Genetics 85, 789-814. 